\title{
Commutative Monoids and Monoid Homomorphism on Lukasiewicz Disjunction and Conjunction Operations Over Pythagorean Fuzzy Matrices
}

\author{
D. Venkatesan ${ }^{1 *}$ and S. Sriram ${ }^{2}$ \\ 'Department of Mathematics, Annamalai University, Annamalainagar - 608002, Tamil Nadu, India; \\ venkat2733327@gmail.com \\ 2Mathematics Wing, Directorate of Distance Education, Annamalai University, Annamalainagar - 608002, \\ Tamil Nadu, India; ssm_3096@yahoo.co.in
}

\begin{abstract}
Objectives: We study some algebraic properties of the operations disjunction $\left(\vee_{L}\right)$ and conjunction $\left(\wedge_{L}\right)$ from Lukasiewicz's type over Pythagorean fuzzy matrices. Methods/Statistical Analysis: We extend these operations of intuitionistic fuzzy matrices to pythagorean fuzzy matrices and proved their algebraic properties. Findings: We discuss some algebraic properties like distributivity, associativity, commutativity, and complementary of these operations. We establish the set of all Pythagorean fuzzy matrices forms a commutative monoid under these operations. Also, we describe a monoid homomorphism over pythagorean fuzzy matrices. Application: Yager constructed the Pythagorean fuzzy decision matrix and its aggregation operators which is used to solve multicriteria decision-making problems.
\end{abstract}

Keywords: Conjunction, Disjunction, Intuitionistic Fuzzy Set, Intuitionistic Fuzzy Matrix, Pythagorean Fuzzy Set, Pythagorean Fuzzy Matrix

\section{Introduction}

The idea of Intuitionistic Fuzzy Matrix (IFM) was introduced by ${ }^{1}$. $\operatorname{In}^{2}$ and ${ }^{\underline{3}}$ to generalize the concept of ${ }^{4}$ fuzzy matrix. Each element in an IFM is expressed by an ordered pair $a_{i j}, a_{i j}^{\prime}$ with $a_{i j}, a_{i j}^{\prime} \in[0,1]$. The sum $a_{i j}+a_{i j}^{\prime}$ of each ordered pairis less than or equal to 1 . Since the appearance of IFM in 2001, many researchers $\frac{5-10}{}$ have importantly contributed for the development of IFM theory and its applications.

Pythagorean Fuzzy Set (PFS) was introduced by ${ }^{11}$. $\mathrm{In}^{12}$, they are discussed some results for PFSs and its algebraic properties. $\operatorname{In}^{\frac{13}{3}}$ studied the PFS and state of the art and future directions. $\operatorname{In}^{\underline{14}}$ introduced Pythagorean Fuzzy Matrix (PFM) and studied its algebraic operations. In $\frac{15}{}$,we defined two new multiplicative operations $\otimes_{1 P}$ and $\otimes_{2 P}$ on PFMs, and investigated their algebraic properties. Also, we discussed the De Morgan's laws, absorption and distributive properties of PFMs.

In ${ }^{16}$ introduced the operations $\vee_{L}$ and $\wedge_{L}$ from Lukasiewicz's type over IFSs. In ${ }^{17}$ introduced two operations $\vee_{L}$ and $\wedge_{L}$ from Lukasiewicz's type over IFMs are studied. $\operatorname{In}^{\frac{18}{6}}$, they are proved the set of all IFMs is a commutative monoid under these operations.

We studied the algebraic properties of two operations $\vee_{L}$ and $\wedge_{L}$ from Lukasiewicz's type over PFMs. Also,

${ }^{*}$ Author for correspondence 
using the relations between $\vee_{L}$ and $\wedge_{L}$ using modal operations.

This study as follows. In Section 2, we will briefly review PFMs and their opertions and Section 3, some properties of the operations $\vee_{L}$ and $\wedge_{L}$ from Lukasiewicz's type over PFMs are studied. The set of all PFMs forms a commutative monoid under these operations. Also, we describe a monoid homomorphism over Pythagorean fuzzy matrices.

\section{Preliminaries}

This section, we will summarize the PFMs and their operations.

Definition 2.1 $1^{14}$ : An PFM is a matrix of pairs $A=\left(a_{i j}, a_{i j}^{\prime}\right)$ of a positive real numbers $a_{i j}, a_{i j}^{\prime} \in[0,1]$ filling the condition $a_{i j}^{2}+a_{i j}^{\prime 2} \leq 1$ for all $\mathrm{i}, \mathrm{j}$.

Definition 2.2 ${ }^{15}$ : For any two PFMs $A, B \in \mathcal{F}_{m n}$,

(i) $A \geq B$ iff $a_{i j} \geq b_{i j}$ and $a_{i j}^{\prime} \leq b_{i j}^{\prime}$.

(ii) $A^{C}=\left(a_{i j}^{\prime} \cdot a_{i j}\right)$.

(iii) $A \vee B=\left(\max \left(a_{i j}, b_{i j}\right), \min \left(a_{i j}^{\prime}, b_{i j}^{\prime}\right)\right)$.

$(i v) A \wedge B=\left(\min \left(a_{i j}, b_{i j}\right), \max \left(a_{i j}^{\prime}, b_{i j}^{\prime}\right)\right)$.

(v) $A X_{1} B=\left(\max \left(a_{i j}, b_{i j}\right), a_{i j}^{\prime} b_{i j}^{\prime}\right)$.

(vi) $A X_{2} B=\left(a_{i j} b_{i j}, \max \left(a_{i j}^{\prime}, b_{i j}^{\prime}\right)\right)$.

(vii) $A \oplus_{P} B=\left(\sqrt{a_{i j}^{2}+b_{i j}^{2}-a_{i j}^{2} b_{i j}^{2}}, a_{i j}^{\prime} b_{i j}^{\prime}\right)$.

(viii) $A \odot_{P} B=\left(a_{i j} b_{i j}, \sqrt{a_{i j}^{\prime 2}+b_{i j}^{\prime 2}-a_{i j}^{\prime 2} b_{i j}^{\prime 2}}\right)$.

$(i x) A \otimes_{1 P} B=\left(\sqrt{\max \left(a_{i j}^{2}, b_{i j}^{2}\right)}, a_{i j}^{\prime} b_{i j}^{\prime}\right)$.

$(x) A \otimes_{2 P} B=\left(a_{i j} b_{i j}, \sqrt{\max \left(a_{i j}^{\prime 2}, b_{i j}^{\prime 2}\right)}\right)$.

(xi) An PFM $O\langle 0,1\rangle$ for all entries is known as the Zero matrix.

An PFM $J\langle 1,0\rangle$ for all entries is known as the Universal matrix.
Definition 2.315: Using intuitionistic fuzzy form of Lukasiewicz implication, we will introduce a disjunction

$$
A \vee_{L} B=\left(\sqrt{\min \left(1, a_{i j}^{2}+b_{i j}^{2}\right)}, \sqrt{\max \left(0, a_{i j}^{\prime 2}+b_{i j}^{\prime 2}-1\right)}\right) .
$$

We will call the new disjunction Lukasiewicz Pythagorean fuzzy disjunction.

Also, we can constract,

$A \wedge_{L} B=\left(\sqrt{\max \left(0, a_{i j}^{2}+b_{i j}^{2}-1\right)}, \sqrt{\min \left(1, a_{i j}^{\prime 2}+b_{i j}^{\prime 2}\right)}\right)$.

We will call the new conjunction Lukasiewicz Pythagorean fuzzy conjunction.

Definition 2.4: A homomorphism between two monoids $(\mathrm{M}, *)$ and $(\mathrm{N},$.$) is a function \mathrm{f}$ from $\mathrm{M}$ to $\mathrm{N}$ such that

(i) $\mathrm{f}\left(\mathrm{x}^{*} \mathrm{y}\right)=\mathrm{f}(\mathrm{x}) \cdot \mathrm{f}(\mathrm{y})$, for all $x, y \in M$.

(ii) $\mathrm{f}\left(\mathrm{e}_{\mathrm{m}}\right)=\mathrm{e}_{\mathrm{n}}$, where $\mathrm{e}_{\mathrm{m}}$ and $\mathrm{e}_{\mathrm{n}}$ are the identites of $\mathrm{M}$ and $\mathrm{N}$ respectively.

\section{Some Results of PFMs}

This section, we introduced the operations $\vee_{L}$ and $\wedge_{L}$ from Lukasiewicz's type over Pythagorean fuzzy matrices. Some results of these operations are studiedwith other predefined operations.

Definition 3.1 :Let $A=\left(a_{i j}, a_{i j}^{\prime}\right)$ be an $m \times n$ PFM. Then we define

(i) $A$ is reflexive iff $\left(a_{i i}, a_{i i}^{\prime}\right)=(1,0)$,

(ii) $A$ s irreflexive iff $\left(a_{i i}, a_{i i}^{\prime}\right)=(0,1)$,

(iii) $A$ is symmetric iff $A^{T}=A$,

(iv) $A$ is nearlyirreflexive if $\left(a_{i i}, a_{i i}^{\prime}\right) \leq\left(a_{i j}, a_{i j}^{\prime}\right)$ for all $i, j$.

Property 3.2 :For any two PFMs $A, B \in \mathcal{F}_{m n}$, we have $(i)$ If $A$ and $B$ are reflexive then $A \vee_{L} B$ and $A \wedge_{L} B$ are reflexive.

(ii) If $A$ and $B$ are irreflexive then $A \vee_{L} B$ and $A \wedge_{L} B$ are irreflexive. 
(iii) If $A$ and $B$ are symmetric then $A \vee B$ and $A \wedge_{L} B$ are symmetric.

(iv) If $A$ and $B$ are nearly irreflexive then $A \vee_{L} B$ and $A \wedge_{L} B$ are nearly irreflexive.

Proof : $(i)$ and $(i i)$ by Definition (2.3).

(iii) Since $A$ and $B$ are symmetric $\left(a_{i j}, a_{i j}^{\prime}\right)=\left(a_{j i}, a_{j i}^{\prime}\right)$,

$$
\left(b_{i j}, b_{i j}^{\prime}\right)=\left(b_{j i}, b_{j i}^{\prime}\right) .
$$

Let $\left(c_{i j}, c_{i j}^{\prime}\right)$ and $\left(d_{i j}, d_{i j}^{\prime}\right)$ are $A \vee_{L} B$ and $A \wedge_{L} B$.

$$
\begin{aligned}
\left(c_{i j},\right. & \left.c_{i j}^{\prime}\right)=\left(\sqrt{\min \left(1, a_{i j}^{2}+b_{i j}^{2}\right)}, \sqrt{\max \left(0, a_{i j}^{\prime 2}+b_{i j}^{\prime 2}-1\right)}\right) \\
& =\left(\sqrt{\min \left(1, a_{j i}^{2}+b_{j i}^{2}\right)}, \sqrt{\max \left(0, a_{j i}^{\prime 2}+b_{j i}^{\prime 2}-1\right)}\right) \\
& =\left(c_{j i}, c_{j i}^{\prime}\right) . \\
& \Rightarrow A \vee_{L} B \text { is symmetric. }
\end{aligned}
$$$$
\text { Similarly we prove }\left(d_{i j}, d_{i j}^{\prime}\right)=\left(d_{j i}, d_{j i}^{\prime}\right) \text {. }
$$$$
\Rightarrow A \wedge_{L} B \text { is symmetric. }
$$

(iv) Since $A$ and $B$ are nearly irreflexive $\left(a_{i i}, a_{i i}^{\prime}\right) \leq\left(a_{i j}, a_{i j}^{\prime}\right)$,

$$
\left(b_{i i}, b_{i i}^{\prime}\right) \leq\left(b_{i j}, b_{i j}^{\prime}\right)
$$

Let $A \vee_{L} B$ is

$$
\begin{aligned}
& \left(c_{i j}, c_{i j}^{\prime}\right)=\left(\sqrt{\min \left(1, a_{i j}^{2}+b_{i j}^{2}\right)}, \sqrt{\max \left(0, a_{i j}^{\prime 2}+b_{i j}^{\prime 2}-1\right)}\right) \\
& \left(c_{i i}, c_{i i}^{\prime}\right)=\left(\sqrt{\min \left(1, a_{i i}^{2}+b_{i i}^{2}\right)}, \sqrt{\max \left(0, a_{i i}^{\prime 2}+b_{i i}^{\prime 2}-1\right)}\right) \\
& a_{i i}^{2} \leq a_{i j}^{2} \text { and } b_{i i}^{2} \leq b_{i j}^{2} \Rightarrow\left(a_{i i}^{2}+b_{i i}^{2}\right) \leq\left(b_{i j}^{2}+a_{i j}^{2}\right) \\
& \Rightarrow \sqrt{\min \left(1, a_{i i}^{2}+b_{i i}^{2}\right)} \leq \sqrt{\min \left(1, a_{i j}^{2}+b_{i j}^{2}\right)}---(3.1)
\end{aligned}
$$

Similarly

$$
\sqrt{\max \left(0, a_{i i}^{\prime 2}+b_{i i}^{\prime 2}-1\right)}>\sqrt{\max \left(0, a_{i j}^{\prime 2}+b_{i j}^{\prime 2}-1\right)}
$$

Above equations (3.1) and (3.2) we get $\left(c_{i j}, c_{i j}^{\prime}\right) \geq\left(c_{i i}, c_{i i}^{\prime}\right)$.

$A \vee_{L} B$ is nearly irreflexive.

$A \wedge_{L} B$ is nearly irreflexive can be proved analogously.

Property 3.3: For any PFM $A \in \mathcal{F}_{m n}$,

(i) $I_{n} \vee_{L}\left(A \vee_{L} A^{T}\right)$ is reflexive.

(ii) $I_{n} \vee_{L}\left(A \vee_{L} A^{T}\right)$ is symmetric.

(iii) $I_{n} \vee_{L}\left(A \vee_{L} A^{T}\right)=I_{n} \vee\left(A \vee_{L} A^{T}\right)$.

Proof:

$$
\begin{aligned}
& \text { Let } A=\left(a_{i j}, a_{i j}^{\prime}\right), A^{T}=\left(a_{j i}, a_{j i}^{\prime}\right) . \\
& \begin{array}{l}
\left(A \vee_{L} A^{T}\right)=\left(\sqrt{\min \left(1, a_{i j}^{2}+a_{j i}^{2}\right)}, \sqrt{\max \left(0, a_{i j}^{\prime 2}+a_{j i}^{\prime}{ }^{2}-1\right)}\right) \\
\quad=\left(s_{i j}, s_{i j}^{\prime}\right) .
\end{array}
\end{aligned}
$$

Let $R=\left(r_{i j}, r_{i j}^{\prime}\right)=I_{n} \vee_{L}\left(A \vee_{L} A^{T}\right)$

$$
=\left\{\begin{array}{l}
\left(\sqrt{\min \left(1,1+s_{i j}\right)}, \sqrt{\max \left(0,0+s_{i j}^{\prime}-1\right)}\right) \text { if } i=j \\
\left(\sqrt{\min \left(1,0+s_{i j}\right)}, \sqrt{\max \left(0,1+s_{i j}^{\prime}-1\right)}\right) \text { if } i \neq j
\end{array}\right.
$$

(i) Suppose that $i=j,\left(r_{i j}, r_{i j}^{\prime}\right)=(1,0)$.

$\Rightarrow R$ is reflexive.

(ii) Suppose that $i \neq j$,

$$
\begin{aligned}
& \left(r_{i j}, r_{i j}^{\prime}\right)=\left(\sqrt{\min \left(1,1+s_{i j}\right)}, \sqrt{\max \left(0,0+s_{i j}^{\prime}-1\right)}\right)=\left(s_{i j}, s_{i j}^{\prime}\right) \\
& =\left(\sqrt{\min \left(1, a_{i j}^{2}+a_{j i}^{2}\right)}, \sqrt{\max \left(0, a_{i j}^{\prime}{ }^{2}+a_{j i}^{\prime}{ }^{2}-1\right)}\right) \\
& =\left(\sqrt{\min \left(1, a_{j i}^{2}+a_{i j}^{2}\right)}, \sqrt{\max \left(0, a_{j i}^{\prime}{ }^{2}+a_{i j}^{\prime 2}-1\right)}\right) \\
& =\left(s_{j i}, s_{j i}^{\prime}\right)=\left(r_{j i}, r_{j i}^{\prime}\right) \\
& \Rightarrow R \text { is symmetric. }
\end{aligned}
$$

(iii) Suppose that $i=j,\left(r_{i i}, r_{i i}^{\prime}\right)=(1,0)$ or 


$$
\left(r_{i j}, r_{i j}^{\prime}\right)=\left(s_{i j}, s_{i j}^{\prime}\right) .
$$

Let

$$
\begin{gathered}
I_{n} \vee\left(A \vee_{L} A^{T}\right)=\left\{\begin{array}{l}
(1,0) \vee\left(s_{i j}, s_{i j}^{\prime}\right)=(1,0) \quad \text { if } i=j \\
(0,1) \vee\left(s_{i j}, s_{i j}^{\prime}\right)=\left(s_{i j}, s_{i j}^{\prime}\right) \text { if } i \neq j
\end{array}\right. \\
\Rightarrow I_{n} \vee_{L}\left(A \vee_{L} A^{T}\right)=I_{n} \vee\left(A \vee_{L} A^{T}\right) .
\end{gathered}
$$

Property 3.4: For any three PFMs $A, B, C \in \mathcal{F}_{m n}$,

$$
A \vee\left(B \vee_{L} C\right)=(A \vee B) \vee_{L}(A \vee C)
$$

\section{Proof :}

Let $\quad\left(d_{i j}, d_{i j}^{\prime}\right)=D, \quad\left(e_{i j}, e_{i j}^{\prime}\right)=E, \quad\left(f_{i j}, f_{i j}^{\prime}\right)=F$ , $\left(g_{i j}, g_{i j}^{\prime}\right)=G$ and $\left(h_{i j}, h_{i j}^{\prime}\right)=H$ are the PFMs of $\left(B \vee_{L} C\right),(A \vee B), \quad(A \vee C), \quad A \vee\left(B \vee_{L} C\right)$ and $(A \vee B) \vee_{L}(A \vee C)$.

Now,

$$
\begin{gathered}
\left(d_{i j}, d_{i j}^{\prime}\right)=\left(\sqrt{\min \left(1, b_{i j}^{2}+c_{i j}^{2}\right)}, \sqrt{\max \left(0, b_{i j}^{\prime 2}+c_{i j}^{\prime 2}-1\right)}\right) \\
\left(e_{i j}, e_{i j}^{\prime}\right)=\left(\max \left(a_{i j}, b_{i j}\right), \min \left(a_{i j}^{\prime}, b_{i j}^{\prime}\right)\right) \\
\left(f_{i j}, f_{i j}^{\prime}\right)=\left(\max \left(a_{i j}, c_{i j}\right), \min \left(a_{i j}^{\prime}, c_{i j}^{\prime}\right)\right) \\
\left(g_{i j}, g_{i j}^{\prime}\right)=\left(\max \left(a_{i j}, d_{i j}\right), \min \left(a_{i j}^{\prime}, d_{i j}^{\prime}\right)\right) \\
\left(h_{i j}, h_{i j}^{\prime}\right)=\left(\sqrt{\min \left(1, e_{i j}^{2}+f_{i j}^{2}\right)}, \sqrt{\max \left(0, e_{i j}^{\prime 2}+f_{i j}^{\prime 2}-1\right)}\right) .
\end{gathered}
$$

We prove $\left(g_{i j}, g_{i j}^{\prime}\right) \leq\left(h_{i j}, h_{i j}^{\prime}\right)$.

That is $g_{i j} \leq h_{i j}, g_{i j}^{\prime}>f_{i j}^{\prime}$.

1. Suppose that $\left(a_{i j}, a_{i j}^{\prime}\right)>\left(b_{i j}, b_{i j}^{\prime}\right),\left(a_{i j}, a_{i j}^{\prime}\right)>\left(c_{i j}, c_{i j}^{\prime}\right)$,

$$
\begin{aligned}
& \left(e_{i j}, e_{i j}^{\prime}\right)=\left(a_{i j}, a_{i j}^{\prime}\right),\left(f_{i j}, f_{i j}^{\prime}\right)=\left(a_{i j}, a_{i j}^{\prime}\right) . \\
& \left(h_{i j}, h_{i j}^{\prime}\right)=\left(\sqrt{\min \left(1,2 a_{i j}^{2}\right)}, \sqrt{\max \left(0,2 a_{i j}^{\prime 2}-1\right)}\right) .
\end{aligned}
$$

1.1 Suppose that $\left(g_{i j}, g_{i j}^{\prime}\right)=\left(a_{i j}, a_{i j}^{\prime}\right) \leq\left(h_{i j}, h_{i j}^{\prime}\right)$.

1.2 Suppose that $\left(g_{i j}, g_{i j}^{\prime}\right)=\left(d_{i j}, d_{i j}^{\prime}\right)$,

$$
\begin{aligned}
& 2 a_{i j}^{2}>\left(b_{i j}^{2}+c_{i j}^{2}\right) \Rightarrow \min \left(1,2 a_{i j}^{2}\right)>\min \left(1, b_{i j}^{2}+c_{i j}^{2}\right) \\
& \Rightarrow h_{i j}>d_{i j}=g_{i j}
\end{aligned}
$$

Similarly $2 a_{i j}^{\prime 2}-1 \leq \max \left(0, b_{i j}^{\prime 2}+c_{i j}^{\prime 2}-1\right)$

$$
\begin{aligned}
& \Rightarrow h_{i j}^{\prime} \leq d_{i j}=g_{i j}^{\prime} . \\
& \left(g_{i j}, g_{i j}^{\prime}\right) \leq\left(h_{i j}, h_{i j}^{\prime}\right) .
\end{aligned}
$$

$\mathbf{1 . 3}$ Suppose that $\left(g_{i j}, g_{i j}^{\prime}\right)=\left(a_{i j}, d_{i j}^{\prime}\right)$.

Then it is true from(1.1) and (1.2).

2. Suppose that $\left(a_{i j}, a_{i j}^{\prime}\right)<\left(b_{i j}, b_{i j}^{\prime}\right),\left(a_{i j}, a_{i j}^{\prime}\right)<\left(c_{i j}, c_{i j}^{\prime}\right)$,

$$
\left(e_{i j}, e_{i j}^{\prime}\right)=\left(b_{i j}, b_{i j}^{\prime}\right),\left(f_{i j}, f_{i j}^{\prime}\right)=\left(c_{i j}, c_{i j}^{\prime}\right) \text {. }
$$

$\left(h_{i j}, h_{i j}^{\prime}\right)=\left(\sqrt{\min \left(1, b_{i j}^{2}+c_{i j}^{2}\right)}, \sqrt{\max \left(0, b_{i j}^{\prime 2}+c_{i j}^{\prime 2}-1\right)}\right)=\left(d_{i j}, d_{i j}^{\prime}\right)$.

2.1 Suppose that $\left(g_{i j}, g_{i j}^{\prime}\right)=\left(a_{i j}, a_{i j}^{\prime}\right)$,

$$
g_{i j}=a_{i j}^{2}<\min \left(1, b_{i j}^{2}+c_{i j}^{2}\right)=h_{i j} \Rightarrow g_{i j}<h_{i j} .
$$

Similarly $a_{i j}^{\prime} \geq h_{i j}^{\prime},\left(g_{i j}, g_{i j}^{\prime}\right) \leq\left(h_{i j}, h_{i j}^{\prime}\right)$.

2.2 Suppose that $\left(g_{i j}, g_{i j}^{\prime}\right)=\left(d_{i j}, d_{i j}^{\prime}\right)=\left(h_{i j}, h_{i j}^{\prime}\right)$. (2.3) and (2.4) trivial from (2.1) and (2.2).

3. Suppose that $\left(a_{i j}, a_{i j}^{\prime}\right)<\left(b_{i j}, b_{i j}^{\prime}\right),\left(a_{i j}, a_{i j}^{\prime}\right)>\left(c_{i j}, c_{i j}^{\prime}\right)$,

$$
\left(h_{i j}, h_{i j}^{\prime}\right)=\left(\sqrt{\min \left(1, b_{i j}^{2}+a_{i j}^{2}\right)}, \sqrt{\max \left(0, b_{i j}^{\prime 2}+a_{i j}^{\prime 2}-1\right)}\right) .
$$

3.1 Suppose that $\left(g_{i j}, g_{i j}^{\prime}\right)=\left(a_{i j}, a_{i j}^{\prime}\right)$,

$$
\min \left(1, b_{i j}^{2}+a_{i j}^{2}\right)>a_{i j}^{2} \Rightarrow h_{i j}>g_{i j} \text { and } b_{i j}^{\prime 2}-1 \leq 0
$$

$$
\begin{aligned}
& \Rightarrow\left(a_{i j}^{\prime 2}+b_{i j}^{\prime 2}-1\right) \leq a_{i j}^{\prime 2} \\
& \Rightarrow \max \left(0, a_{i j}^{\prime 2}+b_{i j}^{\prime 2}-1\right) \leq a_{i j}^{\prime 2} \\
& \Rightarrow h_{i j} \leq g_{i j} .
\end{aligned}
$$

$$
\left(g_{i j}, g_{i j}^{\prime}\right) \leq\left(h_{i j}, h_{i j}^{\prime}\right)
$$


3.2 Suppose that $\left(g_{i j}, g_{i j}^{\prime}\right)=\left(d_{i j}, d_{i j}^{\prime}\right)$,

$a_{i j}^{2}>c_{i j}^{2} \Rightarrow \min \left(1, a_{i j}^{2}+b_{i j}^{2}\right)>\min \left(1, b_{i j}^{2}+c_{i j}^{2}\right) \Rightarrow h_{i j}>d_{i j}=g_{i j}$.

Similarly $h_{i j}^{\prime} \leq d_{i j}^{\prime}=g_{i j}^{\prime} \Rightarrow\left(g_{i j}, g_{i j}^{\prime}\right) \leq\left(h_{i j}, h_{i j}^{\prime}\right)$.

(3.3) and (3.4) trivial from (3.1) and (3.2).

4.Suppose that $\left(a_{i j}, a_{i j}^{\prime}\right)>\left(b_{i j}, b_{i j}^{\prime}\right),\left(a_{i j}, a_{i j}^{\prime}\right)<\left(c_{i j}, c_{i j}^{\prime}\right)$, $\left(h_{i j}, h_{i j}^{\prime}\right)=\left(\sqrt{\min \left(1, a_{i j}^{2}+c_{i j}^{2}\right)}, \sqrt{\max \left(0, a_{i j}^{\prime 2}+c_{i j}^{\prime 2}-1\right)}\right)$.

The proof of (4) similarly to (3).

Hence, from all the above cases we conclude

$$
A \vee\left(B \vee_{L} C\right)=(A \vee B) \vee_{L}(A \vee C) \text {. }
$$

Property 3.5 :For any three PFMs $A, B, C \in \mathcal{F}_{m n}$,

$$
\begin{aligned}
& (i)\left(A \vee_{L} B\right) \vee_{L} C=A \vee_{L}\left(B \vee_{L} C\right) . \\
& (i i)\left(A \wedge_{L} B\right) \wedge_{L} C=A \wedge_{L}\left(B \wedge_{L} C\right) .
\end{aligned}
$$

\section{Proof :}

$(i)$ Let $\left(A \vee_{L} B\right),\left(B \vee_{L} C\right),\left(A \vee_{L} B\right) \vee_{L} C$ and $A \vee_{L}\left(B \vee_{L} C\right)$ are as follows

$\left(d_{i j}, d_{i j}^{\prime}\right)=\left(\sqrt{\min \left(1, a_{i j}^{2}+b_{i j}^{2}\right)}, \sqrt{\max \left(0, a_{i j}^{\prime 2}+b_{i j}^{\prime 2}-1\right)}\right)$

$\left(e_{i j}, e_{i j}^{\prime}\right)=\left(\sqrt{\min \left(1, b_{i j}^{2}+c_{i j}^{2}\right)}, \sqrt{\max \left(0, b_{i j}^{\prime 2}+c_{i j}^{\prime 2}-1\right)}\right)$

$\left(f_{i j}, f_{i j}^{\prime}\right)=\left(\sqrt{\min \left(1, d_{i j}^{2}+c_{i j}^{2}\right)}, \sqrt{\max \left(0, d_{i j}^{\prime 2}+c_{i j}^{\prime 2}-1\right)}\right)$

$\left(g_{i j}, g_{i j}^{\prime}\right)=\left(\sqrt{\min \left(1, a_{i j}^{2}+e_{i j}^{2}\right)}, \sqrt{\max \left(0, a_{i j}^{\prime 2}+e_{i j}^{\prime 2}-1\right)}\right)$

Now we have to prove $\left(f_{i j}, f_{i j}^{\prime}\right)=\left(g_{i j}, g_{i j}^{\prime}\right)$.

1. Suppose that $a_{i j}^{2}+b_{i j}^{2}<1, a_{i j}^{\prime 2}+b_{i j}^{\prime 2}-1 \leq 0$, then $\left(f_{i j}, f_{i j}^{\prime}\right)=\left(\sqrt{\min \left(1, a_{i j}^{2}+b_{i j}^{2}+c_{i j}^{2}\right)}, 0\right)$.

1.1 Suppose that $a_{i j}^{2}+b_{i j}^{2}+c_{i j}^{2}<1, f_{i j}=\left(a_{i j}^{2}+b_{i j}^{2}+c_{i j}^{2}\right)$.
In this case $\left.b_{i j}^{2}+c_{i j}^{2}\right) \leq 1$,

$g_{i j}=\min \left(1, a_{i j}^{2}+b_{i j}^{2}+c_{i j}^{2}\right)=a_{i j}^{2}+b_{i j}^{2}+c_{i j}^{2}=f_{i j}$.

Since $a_{i j}^{\prime 2}+b_{i j}^{\prime 2}-1 \leq 0$,

either $b_{i j}^{\prime 2}+c_{i j}^{\prime 2}-1 \leq 0$ or $>0$.

Suppose $b_{i j}^{\prime 2}+c_{i j}^{\prime 2}-1 \leq 0, g_{i j}^{\prime}=0=f_{i j}^{\prime}$.

Suppose $b_{i j}^{\prime 2}+c_{i j}^{\prime 2}-1>0$,

$g_{i j}^{\prime}=\max \left(0, a_{i j}^{\prime 2}+b_{i j}^{\prime 2}+c_{i j}^{\prime 2}-1-1\right)$

$=\left(b_{i j}^{\prime 2}+c_{i j}^{\prime 2}-1\right)+\max \left(0, c_{i j}^{\prime 2}-1\right)$

$=0=f_{i j}^{\prime}$.

1.2 Suppose that $a_{i j}^{2}+b_{i j}^{2}+c_{i j}^{2}>1,\left(f_{i j}, f_{i j}^{\prime}\right)=(1,0)$.

In this case $b_{i j}^{2}+c_{i j}^{2} \leq 1$ or $>1$.

Suppose that $b_{i j}^{2}+c_{i j}^{2} \leq 1$.

$$
\begin{aligned}
& \left(g_{i j}, g_{i j}^{\prime}\right)=\left(\sqrt{\min \left(1, a_{i j}^{2}+e_{i j}^{2}\right)}, 0\right) \\
& =\left(\sqrt{\min \left(1, a_{i j}^{2}+b_{i j}^{2}+c_{i j}^{2}\right)}, 0\right) \\
& =(1,0) \\
& =\left(f_{i j}, f_{i j}^{\prime}\right) .
\end{aligned}
$$

Suppose that $b_{i j}^{2}+c_{i j}^{2}>1$.

$$
\begin{aligned}
& \left(g_{i j}, g_{i j}^{\prime}\right)=\left(\sqrt{\min \left(1, a_{i j}^{2}+1\right), 0}\right) \\
& =(1,0) \\
& =\left(f_{i j}, f_{i j}^{\prime}\right) .
\end{aligned}
$$

2. Suppose that $a_{i j}^{2}+b_{i j}^{2} \geq 1$,

$a_{i j}^{\prime 2}+b_{i j}^{\prime 2}-1<0,\left(f_{i j}, f_{i j}^{\prime}\right)=(1,0)$.

Since, $a_{i j}^{2}+b_{i j}^{2} \geq 1 \Rightarrow a_{i j}^{2}+b_{i j}^{2}+c_{i j}^{2} \geq 1$,

$b_{i j}^{\prime 2}+c_{i j}^{\prime 2}-1 \leq 0$ or $\geq 0$. 
$\Rightarrow b_{i j}^{2}+c_{i j}^{2} \geq 1$ or $\leq 1, b_{i j}^{\prime 2}+c_{i j}^{\prime 2}-1 \leq 0$ or $\geq 0$.

2.1 Suppose that $b_{i j}^{2}+c_{i j}^{2} \geq 1$,

$b_{i j}^{\prime 2}+c_{i j}^{\prime 2}-1 \leq 0,\left(g_{i j}, g_{i j}^{\prime}\right)=(1,0)$.

2.2 Suppose that $b_{i j}^{2}+c_{i j}^{2} \geq 1, b_{i j}^{\prime 2}+c_{i j}^{\prime 2}-1 \geq 0$,

$$
\begin{aligned}
& \left(g_{i j}, g_{i j}^{\prime}\right)=\left(1, \sqrt{\max \left(0, a_{i j}^{\prime 2}+b_{i j}^{\prime 2}+c_{i j}^{\prime 2}-1-1\right)}\right) \\
& =\left(1, \sqrt{\max \left(0,\left(a_{i j}^{\prime 2}+b_{i j}^{\prime 2}-1\right)+\left(c_{i j}^{\prime 2}-1\right)\right)}\right) \\
& =(1,0) \\
& =\left(f_{i j}, f_{i j}^{\prime}\right) .
\end{aligned}
$$

2.3 Suppose that $b_{i j}^{2}+c_{i j}^{2}<1, b_{i j}^{\prime 2}+c_{i j}^{\prime 2}-1<0$,

$$
\begin{aligned}
& \left(g_{i j}, g_{i j}^{\prime}\right) \\
= & \left(\sqrt{\min \left(1, a_{i j}^{2}+b_{i j}^{2}+c_{i j}^{2}\right)}, \sqrt{\max \left(0, a_{i j}^{\prime 2}+b_{i j}^{\prime 2}+c_{i j}^{\prime 2}-1-1\right)}\right) \\
= & \left(1, \sqrt{\max \left(0,\left(a_{i j}^{\prime 2}+0-1\right)\right)}\right) \\
= & (1,0) \\
= & \left(f_{i j}, f_{i j}^{\prime}\right) .
\end{aligned}
$$

2.4 Suppose that $b_{i j}^{2}+c_{i j}^{2}<1, a_{i j}^{\prime 2}+b_{i j}^{\prime 2}-1>0$.

From(2.2) and (2.3) this is true.

3. Suppose that $a_{i j}^{2}+b_{i j}^{2} \leq 1, b_{i j}^{\prime 2}+c_{i j}^{\prime 2}-1>0$.

$\left(f_{i j}, f_{i j}^{\prime}\right)=\left(\sqrt{\min \left(1, a_{i j}^{2}+b_{i j}^{2}+c_{i j}^{2}\right)}, \sqrt{\max \left(0, a_{i j}^{\prime 2}+b_{i j}^{\prime 2}-1+c_{i j}^{\prime 2}-1\right)}\right)$.

3.1 Suppose that $a_{i j}^{2}+b_{i j}^{2}+c_{i j}^{2}<1$,

$$
a_{i j}^{\prime 2}+b_{i j}^{\prime 2}+c_{i j}^{\prime 2}-2<0 .
$$

$\operatorname{From}(1.1) f_{i j}=g_{i j}$ and $f_{i j}^{\prime}=0$.

Now,

$a_{i j}^{\prime 2}+b_{i j}^{\prime 2}+c_{i j}^{\prime 2}-2=\left(a_{i j}^{\prime 2}-1\right)+\left(b_{i j}^{\prime 2}+c_{i j}^{\prime 2}-1\right)<0$.
Either $b_{i j}^{\prime 2}+c_{i j}^{\prime 2}-1<0$ or $>0$.

Suppose that ${b_{i j}^{\prime}}^{2}+c_{i j}^{\prime 2}-1<0$, then

$$
g_{i j}^{\prime}=\max \left(0, a_{i j}^{\prime 2}+0-1\right)=0=f_{i j}^{\prime} .
$$

Suppose that $b_{i j}^{\prime 2}+c_{i j}^{\prime 2}-1 \geq 0$,

$g_{i j}^{\prime}=\max \left(0, a_{i j}^{\prime 2}+b_{i j}^{\prime 2}+c_{i j}^{\prime 2}-1-1\right)=0=f_{i j}^{\prime}$.

3.2 Suppose that $a_{i j}^{2}+b_{i j}^{2}+c_{i j}^{2}<1$,

$$
\begin{aligned}
& a_{i j}^{\prime 2}+b_{i j}^{\prime 2}+c_{i j}^{\prime 2}-2>0, \\
& f_{i j}=a_{i j}^{2}+b_{i j}^{2}+c_{i j}^{2} \text { from (1.1) } g_{i j}=f_{i j} .
\end{aligned}
$$

$$
\begin{aligned}
f_{i j}^{\prime} & =a_{i j}^{\prime 2}+b_{i j}^{\prime 2}+c_{i j}^{\prime 2}-2=\left(a_{i j}^{\prime 2}-1\right)+\left(b_{i j}^{\prime 2}+c_{i j}^{\prime 2}-1\right) \geq 0 \\
& \Rightarrow b_{i j}^{\prime 2}+c_{i j}^{\prime 2}-1>0 \\
& \Rightarrow g_{i j}^{\prime}=a_{i j}^{\prime 2}+b_{i j}^{\prime 2}+c_{i j}^{\prime 2}-2=f_{i j}^{\prime} .
\end{aligned}
$$

3.3.Suppose that $a_{i j}^{2}+b_{i j}^{2}+c_{i j}^{2}>1$,

$$
a_{i j}^{\prime 2}+b_{i j}^{\prime 2}+c_{i j}^{\prime 2}-2<0 \text {. }
$$

From(1.3) and (3.1) this is true.

3.4 Suppose that $a_{i j}^{2}+b_{i j}^{2}+c_{i j}^{2}>1$,

$$
a_{i j}^{\prime 2}+b_{i j}^{\prime 2}+c_{i j}^{\prime 2}-2>0 \text {. }
$$

From (1.3) and (3.2) this is true.

$$
\left(g_{i j}, g_{i j}^{\prime}\right)=\left(f_{i j}, f_{i j}^{\prime}\right) \text {. }
$$

4. Suppose that $a_{i j}^{2}+b_{i j}^{2} \geq 1, a_{i j}^{\prime 2}+b_{i j}^{\prime 2}-1 \geq 0$.

It is obvious from (2) and (3).

$$
(i i)\left(A \wedge_{L} B\right) \wedge_{L} C=A \wedge_{L}\left(B \wedge_{L} C\right) .
$$

Let

$$
\begin{aligned}
& \left(d_{i j}, d_{i j}^{\prime}\right)=\left(\sqrt{\max \left(0, a_{i j}^{2}+b_{i j}^{2}-1\right)}, \sqrt{\min \left(1, a_{i j}^{\prime 2}+b_{i j}^{\prime 2}\right)}\right) \\
& \left(e_{i j}, e_{i j}^{\prime}\right)=\left(\sqrt{\max \left(0, b_{i j}^{2}+c_{i j}^{2}-1\right)}, \sqrt{\min \left(1, b_{i j}^{\prime 2}+c_{i j}^{\prime 2}\right)}\right)
\end{aligned}
$$


$\left(f_{i j}, f_{i j}^{\prime}\right)=\left(\sqrt{\max \left(0, d_{i j}^{2}+c_{i j}^{2}-1\right)}, \sqrt{\min \left(1, d_{i j}^{\prime 2}+c_{i j}^{\prime 2}\right)}\right)$

$\left(g_{i j}, g_{i j}^{\prime}\right)=\left(\sqrt{\max \left(0, a_{i j}^{2}+e_{i j}^{2}-1\right)}, \sqrt{\min \left(1, a_{i j}^{\prime 2}+e_{i j}^{\prime 2}\right)}\right)$.

We have to Prove $\left(f_{i j}, f_{i j}^{\prime}\right)=\left(g_{i j}, g_{i j}^{\prime}\right)$.

1. Suppose that $a_{i j}^{2}+b_{i j}^{2}-1 \leq 0, a_{i j}^{\prime 2}+b_{i j}^{\prime 2}<1$, then

$$
\left(f_{i j}, f_{i j}^{\prime}\right)=\left(0, \sqrt{\min \left(1, a_{i j}^{\prime 2}+b_{i j}^{\prime 2}+c_{i j}^{\prime 2}\right)}\right) .
$$

\subsection{Suppose that}

$$
a_{i j}^{\prime 2}+b_{i j}^{\prime 2}+c_{i j}^{\prime 2}<1,\left(f_{i j}, f_{i j}^{\prime}\right)=\left(0, \sqrt{\min \left(1, a_{i j}^{\prime 2}+b_{i j}^{\prime 2}+c_{i j}^{\prime 2}\right)}\right)
$$

either $b_{i j}^{\prime 2}+c_{i j}^{\prime 2}<1, b_{i j}^{2}+c_{i j}^{2}-1<0$

$$
\left(g_{i j}, g_{i j}^{\prime}\right)=\left(0, \sqrt{\min \left(1, a_{i j}^{\prime 2}+b_{i j}^{\prime 2}+c_{i j}^{\prime 2}\right)}\right)=\left(f_{i j}, f_{i j}^{\prime}\right) .
$$

Suppose that $b_{i j}^{2}+c_{i j}^{2}-1>0, b_{i j}^{\prime 2}+c_{i j}^{2}<1$,

$$
\begin{aligned}
\left(g_{i j}, g_{i j}^{\prime}\right) & =\left(\sqrt{\max \left(0, a_{i j}^{2}+b_{i j}^{2}+c_{i j}^{2}-2\right)}, \sqrt{\min \left(1, a_{i j}^{\prime 2}+b_{i j}^{\prime 2}+c_{i j}^{\prime 2}\right)}\right) \\
= & \left(0, \sqrt{\min \left(1, a_{i j}^{\prime 2}+b_{i j}^{\prime 2}+c_{i j}^{\prime 2}\right)}\right) \cdot=\left(f_{i j}, f_{i j}^{\prime}\right) .
\end{aligned}
$$

1.2 Suppose that $a_{i j}^{\prime 2}+b_{i j}^{\prime 2}+c_{i j}^{\prime 2}>1$, then

$$
\left(f_{i j}, f_{i j}^{\prime}\right)=(0,1)
$$

$$
\text { Suppose that } b_{i j}^{2}+c_{i j}^{2}-1\left\langle 0, b_{i j}^{\prime 2}+c_{i j}^{\prime 2}\right\rangle 1 \text {. }
$$

$$
\left(g_{i j}, g_{i j}^{\prime}\right)=(0,1)=\left(f_{i j}, f_{i j}^{\prime}\right) \text {. }
$$

Suppose that $b_{i j}^{2}+c_{i j}^{2}-1<0, b_{i j}^{\prime 2}+c_{i j}^{\prime 2}<1$.

$$
\left(g_{i j}, g_{i j}^{\prime}\right)=(0,1)=\left(f_{i j}, f_{i j}^{\prime}\right) \text {. }
$$

Suppose that $b_{i j}^{2}+c_{i j}^{2}-1>0, b_{i j}^{\prime 2}+c_{i j}^{\prime 2}>1$.

$$
\left(g_{i j}, g_{i j}^{\prime}\right)=(0,1)=\left(f_{i j}, f_{i j}^{\prime}\right) \text {. }
$$

Suppose that $b_{i j}^{2}+c_{i j}^{2}-1<0, b_{i j}^{\prime 2}+c_{i j}^{\prime 2}<1$.

$$
\left(g_{i j}, g_{i j}^{\prime}\right)=(0,1)=\left(f_{i j}, f_{i j}^{\prime}\right)
$$

2. Suppose that $a_{i j}^{2}+b_{i j}^{2}-1<0, a_{i j}^{\prime 2}+b_{i j}^{\prime 2} \geq 1$, then $\left(f_{i j}, f_{i j}^{\prime}\right)=(0,1)$.

Clearly it is true from (1.1) and (1.2).

3. Suppose that $a_{i j}^{2}+b_{i j}^{2}-1>0, a_{i j}^{\prime 2}+b_{i j}^{\prime 2} \leq 1$, $\left(f_{i j}, f_{i j}^{\prime}\right)=\left(\sqrt{\max \left(0, a_{i j}^{2}+b_{i j}^{2}+c_{i j}^{2}-2\right)}, \sqrt{\min \left(1, a_{i j}^{\prime 2}+b_{i j}^{\prime 2}+c_{i j}^{\prime 2}\right)}\right)$.

3.1 Suppose that $a_{i j}^{2}+b_{i j}^{2}+c_{i j}^{2}-2<0$,

$$
a_{i j}^{\prime 2}+b_{i j}^{\prime 2}+c_{i j}^{\prime 2}<1
$$

$$
\left(f_{i j}, f_{i j}^{\prime}\right)=\left(0, a_{i j}^{\prime 2}+b_{i j}^{\prime 2}+c_{i j}^{\prime 2}\right) .
$$

For any values of $\left(b_{i j}, b_{i j}^{\prime}\right),\left(c_{i j}, c_{i j}^{\prime}\right)$,

$$
\left(f_{i j}, f_{i j}^{\prime}\right)=\left(g_{i j}, g_{i j}^{\prime}\right)
$$

3.2 Suppose that $a_{i j}^{2}+b_{i j}^{2}+c_{i j}^{2}-2 \geq 0$,

$$
\begin{aligned}
& a_{i j}^{\prime 2}+b_{i j}^{\prime 2}+c_{i j}^{\prime 2}<1, \\
& \left(f_{i j}, f_{i j}^{\prime}\right)=\left(\sqrt{\max \left(0, a_{i j}^{2}+b_{i j}^{2}+c_{i j}^{2}-2\right)}, \sqrt{\min \left(1, a_{i j}^{\prime 2}+b_{i j}^{\prime 2}+c_{i j}^{\prime 2}\right)}\right) . \\
& \text { Since, } a_{i j}^{2}+b_{i j}^{2}+c_{i j}^{2}-2=\left(a_{i j}^{2}-1\right)+\left(b_{i j}^{2}+c_{i j}^{2}-1\right)>0 \\
& \Rightarrow b_{i j}^{2}+c_{i j}^{2}-1 \geq 0 .\left(g_{i j}, g_{i j}^{\prime}\right)=\left(\sqrt{\max \left(0, a_{i j}^{2}+b_{i j}^{2}+c_{i j}^{2}-2\right)}, \sqrt{\min \left(1, a_{i j}^{\prime 2}+b_{i j}^{\prime 2}+c_{i j}^{\prime 2}\right)}\right) \\
& \quad=\left(f_{i j}, f_{i j}^{\prime}\right) .
\end{aligned}
$$

3.3 Suppose that $a_{i j}^{2}+b_{i j}^{2}+c_{i j}^{2}-2<0$,

$$
a_{i j}^{\prime 2}+b_{i j}^{\prime 2}+c_{i j}^{\prime 2}>1
$$

Clearly $f_{i j}=g_{i j}, f_{i j}^{\prime}=1$.

Suppose that $b_{i j}^{\prime 2}+c_{i j}^{\prime 2}>1 \Rightarrow g_{i j}^{\prime}=1$.

Suppose that $b_{i j}^{\prime 2}+c_{i j}^{\prime 2} \leq 1 \Rightarrow g_{i j}^{\prime}=1$.

4. Suppose that $a_{i j}^{2}+b_{i j}^{2}-1>0, a_{i j}^{\prime 2}+b_{i j}^{2} \geq 1$,

$$
\left(f_{i j}, f_{i j}^{\prime}\right)=\left(\sqrt{\max \left(0, a_{i j}^{2}+b_{i j}^{2}+c_{i j}^{2}-2\right)}, 1\right)
$$


From (3) we get $f_{i j}=g_{i j}$.

4.1 Suppose that ${b_{i j}^{\prime}}^{2}+c_{i j}^{2} \geq 1$, then $g_{i j}^{\prime}=1=f_{i j}^{\prime}$ or

Suppose that $b_{i j}^{\prime 2}+c_{i j}^{2}<1$, then $g_{i j}^{\prime}=1=f_{i j}^{\prime}$.

Hence, from all the above cases we conclude

$$
\left(f_{i j}, f_{i j}^{\prime}\right)=\left(g_{i j}, g_{i j}^{\prime}\right) \text {. }
$$

Property 3.6 : For any two PFMs $A, B \in \mathcal{F}_{m n}$,

$$
\begin{aligned}
& (i)\left(A \vee_{L} B\right)^{C} \leq A^{C} \vee_{L} B^{C} . \\
& (i i)\left(A \wedge_{L} B\right)^{C} \geq A^{C} \wedge_{L} B^{C} .
\end{aligned}
$$

Property 3.7: $\mathcal{F}_{m n}$ is the set of all PFMs. Then we have (i) $\left(\mathcal{F}_{m n}, \vee_{L}, O\right)$ is a commutative monoid.

(ii) $\left(\mathcal{F}_{m n}, \wedge_{L}, J\right)$ is a commutative monoid.

\section{Proof:}

By Definition 2.3 and Property 3.5.

Corollary 3.8: For any two monoids $\left(\mathcal{F}_{m n}, \vee_{L}, O\right)$ and $\left(\mathcal{F}_{m n}, \wedge_{L}, J\right)$ and a function $f: \mathcal{F}_{m n} \rightarrow \mathcal{F}_{m n}$ such that $f(A)=A^{C}$, then there exists a monoid homomorphism under $\vee_{L}$ and $\wedge_{L}$ operations.

Proof: Let as consider $A, B \in \mathcal{F}_{m n}$.

$$
\begin{aligned}
f( & \left.A \vee_{L} B\right)=f\left(\sqrt{\min \left(1, a_{i j}^{2}+b_{i j}^{2}\right)}, \sqrt{\max \left(0, a_{i j}^{\prime 2}+b_{i j}^{\prime 2}-1\right)}\right) \\
& =\left(\sqrt{\max \left(0, a_{i j}^{\prime 2}+b_{i j}^{\prime 2}-1\right)}, \sqrt{\min \left(1, a_{i j}^{2}+b_{i j}^{2}\right)}\right) \\
& =\left(\left(a_{i j}^{\prime}, a_{i j}\right) \wedge_{L}\left(b_{i j}^{\prime}, b_{i j}\right)\right) \\
& =A^{C} \wedge_{L} B^{C} \\
& =f(A) \wedge_{L} f(B) . \\
& \text { Hence, } f\left(A \vee_{L} B\right)=f(A) \wedge_{L} f(B) . \\
& f(O)=O^{C}=J, f(J)=J^{C}=O .
\end{aligned}
$$

Definition( 2.4) there exists a monoid homomorphism.

\section{Conclusion}

In this work, we studied some results of two operations and from Luckasiewicz's type over Pythagorean fuzzy matrices. In addition we discussed some properties like distributivity, associativity, commutativity, and complementary of these operations. Also we described amonoid homomorphism over Pythagorean fuzzy matrices.

\section{References}

1. Atanassov KT. Intuitionistic fuzzy set. Fuzzy Sets and System. 1986; 20(1):87-96. https://doi.org/10.1016/S01650114(86)80034-3

2. Khan SK, Pal M, Shyamal AK. Intuitionistic fuzzy matrices. Notes on Intuitionistic Fuzzy Sets. 2002; 8(2):51-62.

3. Im YB, Lee EB, Park SW. The Determinant of square intuitionistic fuzzy matrices. Far East Journal of Mathematical Sciences. 2001; 3(5):789-96.

4. Thomson MG. Convergence of powers of fuzzy matrix. Journal of Mathematical Analysis and Applications. 1977; 57(2):476-80. https://doi.org/10.1016/0022247X(77)90274-8

5. Muthuraji T, Sriram S. Reduction of an intuitionistic fuzzy matrix to fuzzy matrix with some algebraic properties. Annals of Fuzzy Mathematics and Informatics. 2017; 13(4):475-83.

6. Muthuraji T, Sriram S. Representation and decomposition of an intuitionistic fuzzy matrix using some $\left(\alpha, \alpha^{\wedge^{\prime}}\right)$ cuts. Applications and Applied Mathematics. 2017; 12(1):24158.

7. Padder RA, Murugadas P. Max-min operation on intuitionistic Fuzzy matrix. Annals of Fuzzy Mathematics and Informatics. 2016; 12(6):757-66.

8. Padder RA, Murugadas P. Convergence of powers and canonical form of s-transitive intuitionistic fuzzy matrix. New Trends in Mathematical Sciences. 2017; 5(2):229-36. https://doi.org/10.20852/ntmsci.2017.173

9. Padder RA, Murugadas P. Transitivity of generalized intuitionistic fuzzy matrices. New Trends in Mathematical Sciences. 2017; 5(4):73-82. https://doi.org/10.20852/ntmsci.2017.228

10. Padder RA, Murugadas P. On convergence of the max-min composition of intuitionistic fuzzy matrices. International Journal of Pure and Applied Mathematics. 2018; 119(11):233-41.

11. Yager RR. Pythagorean membership grades in multicriteria decision making. IEEE Transactions on Fuzzy Systems. 2014; 22(4):958-65. https://doi.org/10.1109/ TFUZZ.2013.2278989 
12. Peng XD, Yang Y. Some results for pythagorean fuzzy sets. International Journal of Intelligent Systems. 2015; 30(11):1133-60. https://doi.org/10.1002/int.21738

13. Peng X, Selvachandran G. Pythagorean fuzzy set: state of the art and future Directions. Available from: https://link. springer.com/article/10.1007/s10462-017-9596-9

14. Silambarasan I, Sriram S. Algebraic operations on Pythagorean fuzzy matrices. Mathematical Sciences International Research Journal. 2018; 7(2):406-14.

15. Atanassov K, Tcvetkov R. On Lukasiewiczs intuitionistic fuzzy disjunction and conjunction. Annual of Informatics Section, Union of Scientists in Bulgaria. 2010; 3:90-4.
16. Muthuraji T, Sriram S. Some remarks on Lukasiwicz disjunction and conjunction operators on intuitionistic fuzzy matrices. Journal of Advances in Mathematics. 2015; 11(3):5000-6.

17. Muthuraji T, Sriram S. Commutative monoids and monoid homomorphism on Lukasiwicz conjunction and disjunction operators over intuitionistic fuzzy matrices. International Journal of Pure and Engineering Mathematics. 2015; 3(2):63-75. 